\title{
A PRODUÇÃO EM ECONOMIA DA SAÚDE NO BRASIL: ESTUDO BIBLIOMÉTRICO DE 1988-2013
}

\section{Production in health economy in Brazil: bibliometric study 1988-2013.}

Andreza da Silva ${ }^{1}$, Daniela Savi Geremia ${ }^{2}$, Alessandra Regina Müller Germani ${ }^{3}$

${ }^{1}$ Enfermeira graduada pela Universidade Federal da Fronteira Sul (UFFS).

${ }^{2}$ Enfermeira, Doutora em Saúde Coletiva pelo Instituto de Medicina Social da Universidade do Estado do Rio de Janeiro (UERJ), docente dos cursos de Graduação em Enfermagem e Medicina da Universidade Federal da Fronteira Sul (UFFS), Campus de Chapecó-SC. Líder do grupo de pesquisas Políticas Públicas e Gestão em Saúde (PPGS).

${ }^{3}$ Enfermeira, Doutoranda em desenvolvimento e extensão rural pela Universidade Federal de Santa Maria (UFSM), Mestre em Enfermagem pela Universidade Federal de Santa Catarina (UFSC), docente do curso de Graduação em Medicina da Universidade Federal da Fronteira Sul (UFFS), Campus Passo Fundo-RS.

\section{Endereço para correspondência:}

Daniela Savi Geremia

Rua Mato Grosso, 545 E, Bairro Jardim Itália, Chapecó/SC. CEP: 89814-080.

Tel.: (55)84689915

Email: daniela.geremia@uffs.edu.br 


\title{
Resumo
}

A Economia da Saúde (ES) é uma área de conhecimento que tem apresentado nas últimas décadas importantes avanços para a melhoria organizacional e estrutural da gestão pública do Sistema Único de Saúde (SUS). Este estudo bibliométrico teve como objetivo analisar as características da produção de artigos científicos relacionados à ES publicados no período de 1988 a 2013 nas bases de dados SCIELO, LILACS e MEDLINE, Brasil. Foram utilizados os operadores booleanos and e or no cruzamento de palavras-chave, escolhidas a partir dos cinco eixos centrais de pesquisa: a) Saúde e Desenvolvimento Econômico; b) Regulação Econômica em Saúde; c) Alocação de Recursos; d) Eficiência das ações e Serviços de Saúde; e, e) Financiamento das Ações e Serviços de Saúde, estas também foram utilizadas para categorização e análise. A amostra analisada contou com um total de 82 artigos. Os resultados apontam as fragilidades na produção científica, principalmente no eixo $\mathrm{B} \mathrm{e}$ $\mathrm{D}$, e apresentaram predominância nos eixos $\mathrm{C}$ e $\mathrm{E}$, os periódicos de maior publicação foram aqueles com maior qualificação na Saúde Coletiva. Há predomínio nas publicações provenientes da região Sudeste. A ES representa uma área interdisciplinar que carece de políticas de incentivo em pesquisas que desenvolvam novos conhecimentos e práticas de gestão pública do SUS e estimulem o processo de consolidação desta área de conhecimento.

Palavras-chave: Economia da Saúde; Bibliometria; Saúde Pública.

\begin{abstract}
The Health Economics (HE) is an area of knowledge that has shown in the last decades important advances for organizational and structural improvement of public management of the Unified Health System (UHS). This bibliometric study aimed to analyze the scientific papers production traits related to HE published in the period 1988-2013 in SCIELO databases, LILACS and MEDLINE, Brazil. Were used the boolean operators and or at the intersection of keywords, chosen from the five central themes of research: a) Health and Economic Development; b) Economic Regulation in Health; c) Resource Allocation; d) Efficiency of actions and health services; and, e) Financing Shares and Health Services, these were also used for categorization and analysis. The analyzed sample counted with a total of 82 articles. The results show
\end{abstract}




\section{Gestão em Saúde}

weaknesses in scientific production, especially in the axis $B$ and $D$ and showed dominance on axles $\mathrm{C}$ and $\mathrm{E}$, the periodics with largest publication were those with higher qualification in Public Health. There is a predominance in publications from the Southeast region. The HE represents an interdisciplinary area that lacks policies encouragement in research to develop new knowledge and public management practices of the NHS and stimulate the process of consolidation of this area of knowledge.

Keywords: Health Economics; Bibliometrics; Public Health.

\section{INTRODUÇÃO}

O Sistema Único de Saúde (SUS) foi criado pela Constituição Federal em 1988 (CF/88) tendo como uma das diretrizes organizacionais a descentralização do sistema. Esta diretriz tem como intuito aproximar os serviços e ações em saúde da população e a implementação de políticas que atendam as necessidades locoregionais, a partir da ênfase dada ao processo de municipalização ocorrido pós CF/88. Como forma de organizar o SUS e garantir o direito à saúde, foram atribuídos aos municípios, estados e União, competências administrativas, gerenciais e financeiras frente à assistência à saúde da população 4 .

A partir da política de descentralização do SUS as propostas e expectativas eram de que os princípios de universalidade, equidade e integralidade fossem cumpridos e garantissem a acessibilidade universal de toda população à saúde. Entretanto, Geremia ${ }^{14}$, apesar de apontar os inegáveis avanços do sistema, sinaliza que os ideais não foram concretizados plenamente por problemas estruturais, políticos e econômicos, devido principalmente à heterogeneidade e desigualdade existente entre os entes governamentais.

Entre os desafios para a garantia do direito à saúde e consolidação dos princípios do SUS estão a necessidade de um adequado financiamento e alocação dos recursos disponíveis, sendo fomentados pela responsabilidade tripartite e qualificados com a avaliação e utilização de instrumentos para a efetivação de estratégias de ações. Andrade et al. ${ }^{2}$ apontam que a demanda por mais eficiência e maior eficácia da gestão em saúde "[...] estabelece a necessidade e a urgência do 


\section{Gestão em Saúde}

desenvolvimento e consolidação do campo da ES no país, no sentido de produzir estudos e pesquisas que contribuam para a consecução de tais finalidades".

O financiamento, a alocação de recursos financeiros, a avaliação dos serviços, o desenvolvimento do sistema e a eficiência em saúde são temas de grande relevância e representam desafios a serem enfrentados dentro da área da saúde coletiva e consequentemente da ES. Esses temas precisam estar intrinsicamente articulados na formulação e implementação das políticas de saúde, tendo em vista a organização e adequado funcionamento das estruturas e serviços públicos de saúde.

A ES é definida como:

O ramo do conhecimento que tem por objetivo a otimização das ações de saúde, ou seja, o estudo das condições ótimas de distribuição dos recursos disponíveis para assegurar à população a melhor assistência à saúde e o melhor estado de saúde possível, tendo em conta meios e recursos limitados ${ }^{11}$.

Os cinco eixos centrais de pesquisa que sustentam a grande área dos estudos da ES, segundo o Ministério da Saúde ${ }^{5}$, são: 1) Saúde e Desenvolvimento Econômico; 2) Regulação Econômica em Saúde; 3) Alocação de Recursos; 4) Eficiência das ações e Serviços de Saúde; e, 5) Financiamento das Ações e Serviços de Saúde (Figura 1).

Figura 1. Eixos de pesquisa em Economia da Saúde.

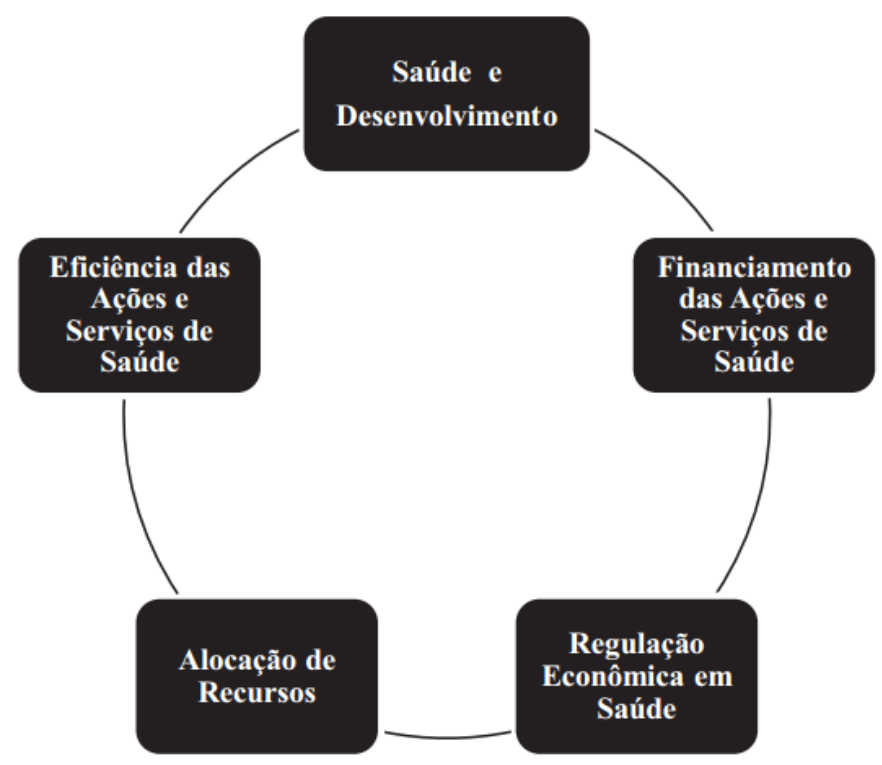

Fonte: BRASIL, 2012, p. 10. 
A ES e seus avanços acarretam diretamente nos processos de tomada de decisões dos gestores e na definição de políticas de saúde e bem-estar da população. Esse é um ramo do conhecimento que conta com poucos profissionais capacitados para trabalhar na gestão em saúde pública, os autores evidenciam ainda que há necessidade de ampliar os debates, as práticas e reforçar um olhar diversificado na área ${ }^{2}$.

O conhecimento em nível científico do que está sendo produzido no Brasil no âmbito da ES leva à ampliação das perspectivas no processo histórico de construção e desenvolvimento dessa área. A ES demanda uma integração interdisciplinar, uma vez que une duas disciplinas de conhecimento distintas, a saúde e a economia. E, apesar desta área do conhecimento ser explorada pelos pesquisadores e gestores desde a década de 80, inclusive tendo criado em 1991 a Associação Brasileira de Economia da Saúde (ABRES), foi somente em 2003 que o Ministério da Saúde (MS) criou o Departamento de Economia da Saúde.

Atualmente, o MS tem afirmado a importância destes conhecimentos, pois cabe ao Estado garantir os direitos constitucionais e isso só é possível quando os gestores públicos enfrentam os problemas organizacionais e estruturais do SUS. As necessidades de saúde aumentam conforme o crescimento populacional e as necessidades básicas da população ${ }^{8}$. Nesse sentido faz-se necessário que a gestão da saúde seja realizada com qualidade, atendendo as demandas e as necessidades para garantia do direito à saúde.

Tendo como fundamento as premissas de integração interdisciplinar e a busca por conhecer novas áreas de conhecimento que desenvolvam estudos direcionados à melhoria do SUS, alguns questionamentos são levantados: Quais são os principais temas abordados nos estudos brasileiros em economia da saúde? Quais são as principais revistas de publicação? Como estão distribuídas as publicações entre as regiões geográficas brasileiras?

Este estudo justifica-se pela importância da área da ES frente à gestão do SUS. A maioria dos estudos em ES são elaborados por profissionais das ciências da saúde. Dentro desta área, apresentam a seguinte composição: "medicina (45\% dos pesquisadores), farmácia (29\%), odontologia (14\%), enfermagem (8\%), fisioterapia ( $2 \%$ e nutrição ( $2 \%)^{\prime 2}$. Pode-se observar que em comparação com a primeira área de maior publicação, a enfermagem, a fisioterapia e a nutrição ainda tem um grande 
campo de crescimento de produção científica a ser explorado, mas, deve-se reforçar que esta temática tem caráter multidisciplinar e para alguns estudos é imprescindível o suporte de outras áreas de conhecimento. Assim, o objetivo deste estudo foi analisar as características da produção de artigos científicos relacionados à ES e seus cinco eixos centrais de pesquisa no período entre 1988 e 2013.

\section{METODOLOGIA}

Esta pesquisa caracteriza-se como bibliométrica, com abordagem quantitativa e descritiva da produção brasileira de artigos científicos. Por bibliometria entende-se "a técnica quantitativa e estatística de medição dos índices de produção e disseminação do conhecimento científico"3, justificando-se assim a abordagem desta pesquisa.

Com base nas questões problema deste estudo, algumas hipóteses foram delimitadas: Acredita-se que a ES é uma área do conhecimento que apresenta pouca produtividade científica; concentração de publicações sem diversificação de revistas; alta concentração de estudos realizados na região sudeste do Brasil e um possível aumento da produção de artigos a partir da criação do Departamento de Economia da Saúde em 2003.

Esta pesquisa buscou analisar a produção de artigos científicos relacionados à ES e seus cinco eixos centrais de pesquisa, conforme apresentados acima. $O$ corte temporal de 1988 a 2013 foi definido por abranger o período pós CF/88, com a criação do SUS, até 2013, o período mais atual possível de identificação e levantamento dos artigos publicados.

Optou-se por fazer o levantamento apenas de artigos publicados em revistas científicas indexadas nas seguintes bases de dados: SCIELO, LILACS e MEDLINE. As três bases de dados incorporam produções de conhecimento de diversas áreas de pesquisa. Fornecem assim informações atualizadas, precisas e confiáveis dos avanços técnico-científicos na forma de periódicos, artigos, teses e afins, de acordo com a demanda do público de acesso.

Na busca por traçar um panorama da produção científica sobre ES algumas etapas foram seguidas: Definição dos critérios de inclusão e exclusão dos estudos; Seleção da amostra realizada no pré-teste; Armazenamento do universo de artigos 


\section{Gestão em Saúde}

coletados no banco de dados do Microsoft Excel; Inclusão dos estudos selecionados em formato de tabela construída a partir do Microsoft Excel; e a discussão e análise dos resultados encontrados.

A partir da caracterização do estudo, foram delimitadas as estratégias e o planejamento para a coleta de dados, categorização dos eixos, quantificação e análise. Os cinco eixos da ES foram adotados como categorias para organização da coleta de dados e estrutura de análise. Portanto, os artigos coletados foram organizados por categoria desde o início da coleta. A elaboração do projeto de estudo ocorreu entre os meses de abril a julho de 2014 e seu desenvolvimento e a coleta de dados no período de agosto a novembro do mesmo ano.

Inicialmente foi realizado um pré-teste para verificação e escolha dos descritores em saúde que melhor representavam os quantitativos das produções em ES em seus diferentes eixos. Sendo desenvolvida uma busca de descritores em artigos científicos encontrados na Biblioteca Virtual da Saúde (BVS). Em seguida foram realizados diversos cruzamentos de palavras utilizando operadores booleanos and ou or, no intuito de elencar os melhores indicadores para este estudo.

Com a avaliação positiva do desenvolvimento do pré-teste na busca por descritores e variáveis que contemplassem o maior número possível de publicações disponíveis para o objetivo do estudo, verificando o esgotamento de possibilidades de busca pela repetição dos resultados, foi realizada a etapa de coleta de dados do estudo bibliométrico.

Para a coleta de dados na SCIELO, LILACS e MEDLINE utilizou-se os operadores booleanos and e/ou or cruzando as palavras que nomeiam os eixos de pesquisa. Na SCIELO a busca foi desenvolvida no site <http://www.scielo.br/>, no formulário básico de busca por artigos, na LILACS e MEDLINE no portal da BVS no site <http://bvsalud.org/>, em busca avançada. Para cada um dos cinco eixos foram desenvolvidas buscas diferentes com base na avaliação e seleção dos descritores de maior alcance. As publicações coletadas nos cinco eixos da ES totalizaram 339 (trezentos e trinta e nove) artigos científicos, estes foram submetidos aos critérios de inclusão e exclusão para análise.

Os critérios de inclusão para análise foram: a) $O$ artigo ter relação com um dos cinco eixos da ES; b) Publicação dentro do período de corte temporal de 19882013; c) Disponibilidade em língua portuguesa; d) Caracterizar-se como artigo 


\section{Gestão em Saúde}

científico. E como critérios de exclusão, utilizou-se: a) Artigos não relacionados ao tema da ES; b) Artigos repetidos no mesmo eixo; c) Entrevistas ou notas de editor. Com base nos critérios citados, foram excluídos do estudo 257 (duzentos e cinquenta e sete) artigos. Deste modo o estudo contou como amostra para análise um total de 82 (oitenta e dois artigos) proveniente de 27 periódicos científicos.

Os artigos selecionados foram organizados para análise bibliométrica em tabela no Microsoft Excel de acordo com o tema de cada eixo a partir das seguintes variáveis bibliométricas: título, nome dos autores, resumo, periódicos de publicação, ano de publicação e região geográfica da publicação identificada a partir das instituições de filiação dos autores. A seguir são apresentados os principais resultados da análise dos dados coletados.

\section{RESULTADOS E DISCUSSÃO}

Ao iniciar a análise dos principais resultados deste estudo um importante viés de pesquisa precisa ser destacado. Na leitura deste artigo, se deve levar em consideração a existência de artigos científicos publicados sobre o tema da ES que não estão contemplados neste estudo. $O$ motivo se deve ao fato de que muitas revistas não são indexadas nas bases de dados selecionadas para este estudo. Portanto, todos os resultados e análises apresentadas estão relacionados às bases de dados SCIELO, LILACS e MEDLINE.

$\mathrm{Na}$ coleta de dados foi observado que os pesquisadores utilizam variadas práticas de citação e indicadores confusos ou errôneos para descrever/caracterizar suas produções, não retratando fidedignamente o assunto principal do artigo. Fato esse já apresentado por Del Nero'11, que afirma não haver escassez de produções relacionadas à ES, mas que a divulgação desse conhecimento não faz jus ao capital intelectual existente.

Para a análise bibliométrica e também para qualificação dos eixos analisados, os resumos dos artigos foram lidos no intuito de identificar quais os principais eixos abordados, buscando compreender a produção brasileira de artigos científicos em ES no período de 1988 a 2013.

A figura 2 apresenta o total de artigos selecionados por eixo e o total entre todos os eixos. Os eixos com maior quantidade de publicação estão relacionados aos 


\section{Gestão em Saúde}

temas Alocação de recursos (eixo 3) e Financiamento das ações e serviços de saúde (eixo 5). A concentração no eixo 3 e 5 pode ser considerada pela crescente ênfase acerca da efetiva avaliação de prioridades e distribuição de recursos financeiros limitados, este é um grupo amplo no qual foram identificados diversos artigos em duplicidade entre os eixos. Estando esse caráter alocativo, distributivo e estabilizador diretamente ligado ao eixo 4, - Eficiência das ações e serviço de saúde - que representa o terceiro eixo com mais publicações encontradas.

Figura 2. Totais dos artigos científicos selecionados para análise no período de 19882013.

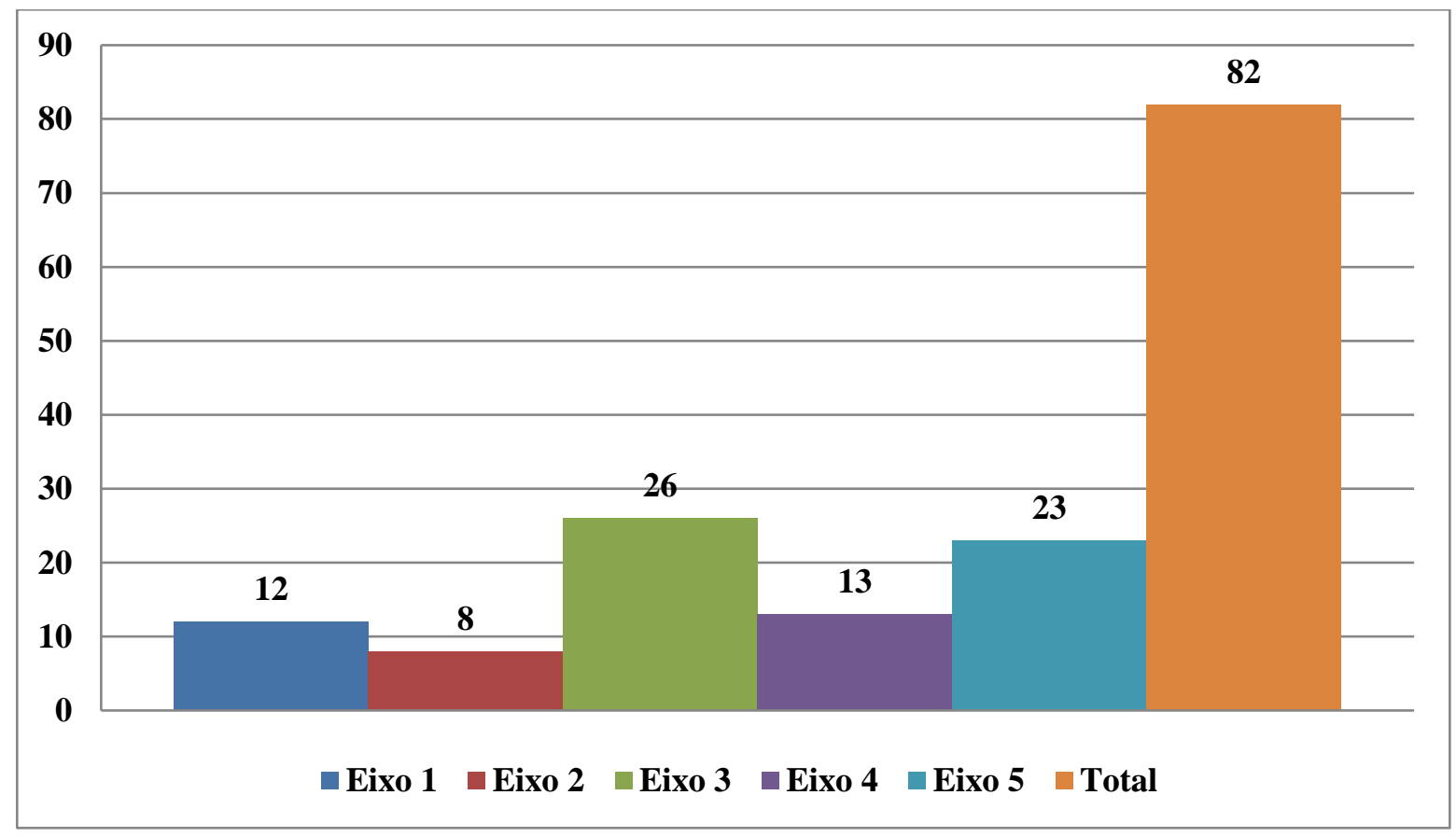

Fonte: Elaboração dos autores (2015).

Entre os anos de 1988 a 1994 nenhum artigo sobre ES foi encontrado a partir do cruzamento dos descritores selecionados. Estudos de Saes ${ }^{17}$ e Andrade et al. ${ }^{2}$, por exemplo, apresentam produção científica na área da ES neste período, mas, no recorte destes estudos foram incluídos diversos tipos de publicações e não apenas artigos científicos, além de mais bases de indexação.

Conforme se observa no Quadro 1, artigos publicados entre os anos 1995 e 2013, foi no ano de 2012 que se atingiu o maior número de publicações selecionadas para análise deste estudo, totalizando 20 artigos, quantidade esta que supera a 


\section{Gestão em Saúde}

produção de 1995 a 2006, mostrando com o estudo a estabilização de publicações na metade da década de 90 e início dos anos 2000. Uma hipótese é que, devido a criação do SUS, temas relacionados a ES passaram a ser prioridades na gestão pública.

Quadro 1. Quantidade de artigos publicados por ano em economia da saúde indexados na base de dados SCIELO, MEDLINE e LILACS, a partir dos descritores selecionados.

\begin{tabular}{|c|c|c|c|c|c|c|c|c|c|c|c|c|c|c|c|c|c|c|c|}
\hline Eixos & 1995 & 1996 & 1997 & 1988 & 1999 & 2000 & 2001 & 2002 & 2003 & 2004 & 2005 & 2006 & 2007 & 2008 & 2009 & 2010 & 2011 & 2012 & 2013 \\
\hline Eixo 1 & 1 & 0 & 0 & 1 & 0 & 0 & 0 & 0 & 0 & 0 & 0 & 0 & 3 & 0 & 0 & 0 & 1 & 6 & 0 \\
\hline Eixo 2 & 0 & 0 & 0 & 0 & 0 & 0 & 0 & 1 & 0 & 0 & 0 & 0 & 0 & 0 & 0 & 0 & 1 & 5 & 1 \\
\hline Eixo 3 & 1 & 0 & 0 & 0 & 0 & 1 & 0 & 1 & 1 & 1 & 4 & 2 & 2 & 1 & 3 & 1 & 4 & 2 & 2 \\
\hline Eixo 4 & 0 & 0 & 0 & 0 & 0 & 0 & 0 & 0 & 0 & 0 & 0 & 1 & 0 & 3 & 2 & 2 & 2 & 2 & 1 \\
\hline Eixo 5 & 1 & 0 & 0 & 0 & 0 & 0 & 0 & 0 & 1 & 0 & 1 & 0 & 2 & 2 & 5 & 0 & 3 & 5 & 3 \\
\hline Total & 3 & 0 & 0 & 1 & 0 & 1 & 0 & 2 & 2 & 1 & 5 & 3 & 7 & 6 & 10 & 3 & 11 & 20 & 7 \\
\hline
\end{tabular}

Fonte: Elaboração dos autores (2015).

Em segundo lugar temos o ano de 2011 com 11 artigos e em terceiro lugar o ano de 2009 com 10 artigos. Entre a relação do ano de maior publicação e o eixo em destaque, temos no ano de 2012 o eixo 1, Saúde e desenvolvimento econômico, com 6 artigos. Em 2011 o eixo 3, Alocação de recursos com 4 artigos e em 2009 o eixo 5, Financiamento das ações e serviços de saúde, com 5 artigos.

Quanto aos periódicos em que os artigos obtiveram maior publicação no corte temporal do estudo, segundo seus eixos, pode-se verificar uma distribuição maior sobre Saúde e desenvolvimento econômico (Eixo 1) na Revista de Saúde Pública, com 6 


\section{Gestão em Saúde}

artigos, e na revista Ciência e Saúde Coletiva, com 4 artigos. Os artigos sobre Regulação econômica em saúde (Eixo 2), foram encontrados em maior quantidade na revista Physis, com 3 artigos, conforme se observa no Quadro 2.

Quadro 2. Distribuição dos artigos científicos por periódico de publicação 1988-2013.

\begin{tabular}{|c|c|c|}
\hline Periódicos de publicação & $\begin{array}{c}\text { Quantidade de } \\
\text { artigos } \\
\text { publicados }\end{array}$ & $\begin{array}{l}\text { Percentagem de } \\
\text { artigos por revista }\end{array}$ \\
\hline \multicolumn{3}{|c|}{ Eixo 1 - Saúde e Desenvolvimento econômico } \\
\hline $\begin{array}{c}\text { Revista Brasileira de Engenharia } \\
\text { Biomédica }\end{array}$ & 1 & $8 \%$ \\
\hline Revista de Saúde Pública & 6 & $50 \%$ \\
\hline Ciência e Saúde Coletiva & 4 & $33 \%$ \\
\hline Saúde e Sociedade & 1 & $8 \%$ \\
\hline Total & 12 & $100 \%$ \\
\hline \multicolumn{3}{|c|}{ Eixo 2 - Regulação econômica em saúde } \\
\hline Saúde Debate & 1 & $13 \%$ \\
\hline Ciência e Saúde Coletiva & 1 & $13 \%$ \\
\hline Physis & 3 & $38 \%$ \\
\hline Epidemiologia e Serviços de Saúde & 1 & $13 \%$ \\
\hline Revista de Saúde Pública & 1 & $13 \%$ \\
\hline Sociologias & 1 & $13 \%$ \\
\hline Total & 8 & $100 \%$ \\
\hline \multicolumn{3}{|c|}{ Eixo 3 - Alocação de recursos } \\
\hline Ciência e Saúde Coletiva & 6 & $23 \%$ \\
\hline Texto Contexto - Enfermagem & 2 & $8 \%$ \\
\hline Revista Saúde Pública & 6 & $23 \%$ \\
\hline Cadernos de Saúde Pública & 4 & $15 \%$ \\
\hline Revista Brasileira de Enfermagem & 1 & $4 \%$ \\
\hline $\begin{array}{l}\text { Revista da Escola de Enfermagem da } \\
\text { USP }\end{array}$ & 2 & $8 \%$ \\
\hline
\end{tabular}




\section{Gestão em Saúde}

\begin{tabular}{|c|c|c|}
\hline Periódicos de publicação & $\begin{array}{c}\text { Quantidade de } \\
\text { artigos } \\
\text { publicados }\end{array}$ & $\begin{array}{l}\text { Percentagem de } \\
\text { artigos por revista }\end{array}$ \\
\hline Saúde Sociedade & 2 & $8 \%$ \\
\hline $\begin{array}{c}\text { Revista Latino Americana de } \\
\text { Enfermagem }\end{array}$ & 1 & $4 \%$ \\
\hline Saúde e Sociedade & 1 & $4 \%$ \\
\hline Physis & 1 & $4 \%$ \\
\hline Total & 26 & $100 \%$ \\
\hline \multicolumn{3}{|c|}{ Eixo 4 - Eficiência das ações e serviços de saúde } \\
\hline Physis & 4 & $31 \%$ \\
\hline $\begin{array}{c}\text { Arquivos Brasileiros de Endocrinologia } \\
\text { e Metabologia }\end{array}$ & 1 & $8 \%$ \\
\hline Cadernos de Saúde Pública & 1 & $8 \%$ \\
\hline Saúde e Sociedade & 1 & $8 \%$ \\
\hline Revista Associação Médica Brasileira & 1 & $8 \%$ \\
\hline Revista de Administração Pública & 1 & $8 \%$ \\
\hline Revista Brasileira de Medicina & 1 & $8 \%$ \\
\hline Einstein (São Paulo) & 1 & $8 \%$ \\
\hline Arquivos de Gastroenterologia & 1 & $8 \%$ \\
\hline RGO - Revista Gaúcha de Odontologia & 1 & $8 \%$ \\
\hline Total & 13 & $100 \%$ \\
\hline \multicolumn{3}{|c|}{ Eixo 5 - Financiamento das ações e serviços de saúde } \\
\hline Economia e Sociedade & 1 & $4 \%$ \\
\hline Saúde e Sociedade & 3 & $13 \%$ \\
\hline Ciência e Saúde Coletiva & 5 & $22 \%$ \\
\hline Revista de Saúde Pública & 1 & $4 \%$ \\
\hline Cadernos de Saúde Pública & 8 & $35 \%$ \\
\hline Serviço Social \& Sociedade & 1 & $4 \%$ \\
\hline Trab. Educ. Saúde & 2 & $9 \%$ \\
\hline Textos e Contextos (Porto Alegre) & 1 & $4 \%$ \\
\hline Saúde Debate & 1 & $4 \%$ \\
\hline
\end{tabular}




\section{Gestão em Saúde}

\begin{tabular}{|c|c|c|}
\hline Periódicos de publicação & $\begin{array}{c}\text { Quantidade de } \\
\text { artigos } \\
\text { publicados }\end{array}$ & $\begin{array}{c}\text { Percentagem de } \\
\text { artigos por revista }\end{array}$ \\
\hline Total & 23 & $100 \%$ \\
\hline
\end{tabular}

Fonte: Elaboração dos autores (2015).

Já os artigos relacionados à Alocação de recursos (Eixo 3), foram encontrados em maior quantidade na Revista de Saúde Pública e Ciência e Saúde Coletiva, com 6 artigos em cada, e na revista Cadernos de Saúde Pública, com 4 artigos publicados.

Quanto a Eficiência das ações e serviços de saúde (Eixo 4), foram encontrados 4 artigos na revista Physis e 1 nas demais. No Financiamento das ações e serviços de saúde (Eixo 5), foram encontrados 8 artigos na revista Cadernos de Saúde Pública e 5 na Revista Ciência e Saúde Coletiva.

A partir das instituições de filiação dos autores, foi identificada a distribuição geográfica dos artigos segundo os eixos da ES, sendo descrita a seguir:

\section{Eixo 1 - Saúde e Desenvolvimento}

No Eixo 1 obteve-se para análise o total de 12 artigos sobre Saúde e Desenvolvimento Econômico, sendo que destes, 9 foram do estado do Rio de Janeiro, 2 de São Paulo e 1 da Paraíba. Do total de artigos, 6 deles têm em comum dois autores que se repetem, o que gera a hipótese de que foram produzidos pelo mesmo grupo de pesquisas.

Andrade et. al. ${ }^{2}$ identificaram 48 grupos de pesquisa do CNPq cujos trabalhos estão relacionados às áreas temáticas referentes à ES. Sendo dos 15.158 grupos de pesquisa avaliados, 4.914 (32,4\%) atuantes na área de saúde e destes, 1\% na área de ES, $0,3 \%$ do total de grupos dessa base. Além da concentração dos grupos de estudos voltados a ES em poucas áreas geográficas do país.

Percebe-se, através das publicações encontradas no eixo 1, a necessidade da ampliação e disseminação geográfica de estudos científicos sobre 0 desenvolvimento econômico da saúde. Tem-se o desafio do desenvolvimento frente aos diferentes interesses sociais, políticos e econômicos, necessitando estratégias políticas para estreitar a relação complexa entre a saúde e o desenvolvimento ${ }^{7,12}$. 
Como proposta de intervenção no desenvolvimento econômico da saúde, Dain ${ }^{10}$ aborda a estratégia de longo prazo vinculada às demandas do gasto público e a importância dos instrumentos de financiamento, bem como o avanço tecnológico do Brasil. Sendo a inovação em saúde presente em grande parte das temáticas apresentadas nas publicações.

A inovação é responsável por uma parcela importante do investimento nacional em pesquisa e desenvolvimento, possuindo uma base industrial e de serviços que articula tecnologias portadoras de futuro, com o protagonismo da saúde na geração de inovação?

Das 12 publicações analisadas, tem-se predominantemente o caráter do Estado nas estratégias de ações e como principal protagonista do desenvolvimento econômico da saúde, principalmente no financiamento dos recursos. Também abordam os desafios para o alcance de avanços no desenvolvimento da ES, os quais remetem ao bem-estar social e efetivação do sistema público de saúde.

\section{Eixo 2 - Regulação Econômica em Saúde}

No eixo 2, totalizou-se para análise 8 artigos sobre Regulação econômica em saúde, sendo que destes 3 foram do estado do Rio de Janeiro, 2 de São Paulo, 2 do Rio Grande do Sul e 1 de Pernambuco. Sem haver repetição de autores no total de artigos.

Tendo os artigos uma apresentação ampla quanto à conceituação da regulação em saúde, podemos considerar Santos e Merhy ${ }^{18}$ quando referem-se à regulação em dois grandes eixos:

A macrorregulação em saúde, como processo de formulação e implementação das políticas pública nesse setor; relações entre os setores público e privado; fontes e ações de financiamento da política; a contratação, formação e remuneração dos trabalhadores da saúde; a intersetorialidade e o controle social. E a microrregulação abrangendo as práticas de assistência à saúde.

Os artigos selecionados estão relacionados à regulação dos serviços de saúde, abordando em sua maioria na microrregulação ações e serviços assistenciais dos diferentes níveis de saúde e na macrorregulação no que se refere às políticas públicas em saúde. 
As publicações também ressaltam a responsabilização tripartite às fragilidades nas regulações já pactuadas. Fragilidades as quais são expostas em práticas regulamentadoras pouco efetivas ${ }^{13}$.

As questões abordadas nos artigos não são menos importantes, mas percebe-se a necessidade de estudos com perspectivas econômicas. Estando a regulação econômica em saúde ligada a garantia do art. 196 da CF/88, um marco regulatório mediante as políticas sociais e econômicas, bem como na efetivação dos princípios e diretrizes do SUS.

\section{Eixo 3 - Alocação de Recursos}

No Eixo 3 obteve-se para análise um total de 26 artigos sobre Alocação de recursos, sendo que destes 12 foram do estado de São Paulo, 4 do Rio de Janeiro, 2 do Rio Grande do Sul, 2 de Pernambuco, 1 de Minas Gerais, 1 do Mato Grosso, 1 de Brasília, 1 do Piauí, 1 do Paraná e 1 de Santa Catarina.

Grande parte das publicações refere-se à necessidade de alocação de recursos nos serviços hospitalares e para ampla demanda de medicamentos. Sendo sinalizada a ineficiência nas aplicações de recursos financeiros para a aquisição de medicamentos, com o aumento considerável de gastos públicos ${ }^{6}$.

Percebe-se o entendimento de que os recursos são escassos e a necessidade de estratégias de controle para a otimização da equidade na distribuição e alocação desses recursos. A equidade vem em confronto com a concentração desproporcional de recursos e a desarticulação entre os serviços, agravados pela diversidade sociocultural, desigualdade socioeconômica e singularidade epidemiológica que distinguem as regiões brasileiras ${ }^{16}$.

Quanto à gestão e planejamento na alocação de recursos, abordados nas publicações, foi percebido que há grande enfoque na priorização do custo-utilidade ou custo-benefício, e não na eficiência, eficácia e efetividade. Tendência esta apontada por Ugá ${ }^{21}$ em seu estudo com base internacional, referindo resultados positivos sobre a saúde da população pela adoção de sistemas de alocação de recursos diferenciados, com foco no custo-efetividade dos recursos destinados aos serviços de saúde. Possibilitando o desenvolvimento de pesquisas nesse âmbito. 


\section{Eixo 4 - Eficiência das Ações e Serviços de Saúde}

O Eixo 4 obteve a análise dos 13 artigos selecionados, referentes a Eficiência das ações e serviços de saúde. Sendo que destes 5 foram do estado de São Paulo, 2 do Rio de Janeiro, 1 do Amazonas, 1 de Brasília, 1 do Paraná, 1 do Mato Grosso, 1 de Minas Gerais e 1 de Santa Catarina. Do total de artigos não houve repetição de autores.

Na quantidade escassa de estudos encontrados sobre a eficiência das ações e serviços em saúde voltados a economia, percebeu-se à predominância de publicações atuais relacionadas ao custo-efetividade, em sua maioria análises a nível hospitalar e/ou no modelo curativista com ênfase no tratamento da saúde e na medicalização. Também quanto à avaliação da eficiência da utilização de diferentes vacinas na prevenção de agravos.

Observando-se que Marinho ${ }^{15}$ aponta a eficiência como um atributo avaliativo da qualidade das ações e serviços do SUS, aponta-se a necessidade de futuros estudos para além das abordagens curativista e hospitalocêntrica, que, segundo Zucchi ${ }^{20}$, demandam maior gasto econômico na saúde.

A gestão de custos visando à eficiência possibilita não somente gastar menos, mas principalmente o melhor gasto, possibilitando, assim:

[...] uma visão mais acurada e ampliada da realidade financeira, já que permite olhar por outro ângulo como são gastos os recursos disponíveis, permitindo identificar os exageros e destinar os recursos na quantidade certa para serem aplicados nas atividades mais necessárias ${ }^{1}$.

A avaliação da eficiência das ações e serviços a nível básico de saúde acarreta no reconhecimento e intervenção das fragilidades para melhoria dos serviços prestados a população. Podendo ser considerada a eficiência na prevenção de agravos e promoção da saúde, um alicerce na ampliação do conceito de eficiência para além da redução de custos, sendo passível o aprofundamento de estudos neste eixo com grande relevância social e econômica.

\section{Eixo 5 - Financiamento das Ações e Serviços de Saúde}

O Eixo 5, referente ao Financiamento das ações e serviços de saúde, obteve em sua distribuição geográfica 23 artigos selecionados, sendo 8 do estado do Rio de 


\section{Gestão em Saúde}

Janeiro, 6 de São Paulo, 3 do Paraná, 2 de Brasília, 1 de Santa Catarina, 1 da Bahia, 1 de Pernambuco e 1 do Rio Grande do Norte.

Os artigos encontrados estão relacionados aos avanços políticos e históricos frente à consolidação do SUS e às estratégias de financiamento do sistema, sendo abordada predominantemente a responsabilidade das três esferas de governo.

No direcionamento do financiamento das ações e serviços em saúde, são abordadas diversas leis e normativas impostas para garantia dos direitos de saúde. Entre elas há a grande presença da Emenda Constitucional no 29/2000 (EC/29-2000) como fator decisório no clareamento das responsabilidades financeiras tripartites ${ }^{10,14}$.

Os autores ainda citam a necessidade de agregar as políticas na consolidação prática e efetiva dos princípios e diretrizes do sistema público de saúde. "[...] embora se tenha avançado nos investimentos em saúde com a EC 29/2000, ainda não se reduziu o déficit social da saúde como política universal e equânime"19.

Frente aos desafios tributários e alocativos no financiamento em saúde, temse a importância do desenvolvimento de pesquisas que possam avaliar e otimizar o financiamento de recursos estáveis e suficientes. Os gestores da saúde, dos diferentes níveis de governo, devem zelar pela qualidade do seu gasto e pela racionalização e redução de seus custos ${ }^{10}$, afirmando ainda que:

A reivindicação do SUS pelo seu direito ao financiamento suficiente e sustentável nada mais é do que a necessidade de honrar seu compromisso junto à população brasileira, materializando a saúde como direito social e garantindo o acesso a e a utilização de serviços. Para tanto, requer que sejam viabilizados, depois de duas décadas, recursos para seu custeio e para investimentos que corrigirão as desigualdades na oferta de infraestrutura de Saúde em todo o território nacional. ${ }^{10}$

Embora a produção científica selecionada no eixo 5 tenha atingido o segundo maior número de produções encontradas por eixo, percebe-se a importância do desenvolvimento de estudos e de resoluções para os diversos empasses do financiamento do SUS. Sendo este um pilar de sustentação econômica para a efetivação do sistema de saúde.

Assim, a precariedade das ações financeiras implica diretamente na alocação de recursos, eficiência, regulação econômica e desenvolvimento, sendo visível a relação dos cinco eixos da ES na garantia de um sistema público de saúde com qualidade. 


\section{CONCLUSÃO}

Ao analisar as características dos artigos produzidos na área da ES, no corte temporal de 1988-2013, foi possível identificar lacunas e fragilidades na produção científica e apontar os principais eixos da ES que necessitam de mais estudos e incentivos.

O eixo 2, referente à Regulação econômica em saúde, obteve menor quantificação de artigos encontrados, com 9,7\%, e o eixo 4, sobre Eficiência das ações e serviços de saúde, com 15,8\%. Em contrapartida, observa-se a predominância nas publicações encontradas no eixo 3 , Alocação de recursos, com $31,7 \%$, e no eixo 5 , Financiamento das ações e serviços de saúde, com $28,0 \%$.

No desenvolvimento de produções no território brasileiro, as regiões geográficas que apontaram os piores indicadores e consequentemente exigem maior necessidade de avanço no desenvolvimento científico neste campo de conhecimento foram: a região norte com $1,21 \%$ e nordeste e centro-oeste com $9,75 \%$ cada. Tendo prevalência de estudos no sudeste, com $64,63 \%$ e no sul do país, com $14,63 \%$ do total de artigos analisados. Este dado também pode ser confirmado devido à concentração dos grupos e incentivos às pesquisas nestas regiões, segundo estudos $^{2}, 64 \%$ estão localizados na região sudeste.

Os principais periódicos de publicação em ES, com 15,85\% das publicações, foram a Cadernos de Saúde Pública, com 14,63\%, a Ciência e Saúde Coletiva, com $9,75 \%$ e a revista Physis. Percebeu-se, através dos artigos coletados no presente estudo, uma estagnação na produção em ES entre 1988 e 1995 e um crescimento instável no período entre 2002 e 2012, de acordo com as bases de indexação analisadas.

Os periódicos da base SCIELO, LILACS e MEDLINE nos permitem analisar os indicadores como fator de impacto, ineditismo e originalidade, inovações científicas e tecnológicas e sua aplicabilidade pelos gestores. O que se observa é que as revistas com maior publicação na área são qualificadas pela Coordenação de Aperfeiçoamento de Pessoal de Nível Superior (CAPES) com Qualis A2 para a área saúde coletiva e Qualis B1 para área interdisciplinar.

A bibliometria como instrumento de avaliação do tamanho, crescimento e distribuição das produções científicas em ES contribui diretamente na análise e 


\section{Gestão em Saúde}

desenvolvimento das necessidades de estudo, caracterizando a realidade econômica do sistema de saúde. Sendo sua utilização um meio de otimização das decisões e estratégias de ações, entre elas as políticas de saúde, bem como a efetiva alocação de recursos para melhoria dos serviços de saúde.

Os indicadores bibliométricos permitem medir a produção acadêmica com base nos dados da literatura científica, mas, na leitura dos estudos com a utilização deste método é necessário cautela e maior atenção aos cruzamentos de descritores e critérios de inclusão e exclusão dos estudos para compreender o recorte dado pelo autor e não incorrer no risco de realizar análises precipitadas. Inclusive é válido destacar que muitos profissionais de saúde estudam procedimentos e tecnologias do ponto de vista econômico e utilizam os descritores e palavras-chave que melhor thes concerne, resultando na dificuldade de identificar a produção de artigos através de busca nas bases de dados.

Não se pretendeu avaliar a qualidade das produções científicas na área, mas compreender as lacunas de publicação de artigos dentro das subáreas de divisão (eixos) da ES. O olhar crítico para este estudo está centrado no estímulo às iniciativas de estudos interdisciplinares.

As mudanças organizacionais dos serviços de saúde a partir da institucionalização do SUS, que pretende a universalização do acesso, apresentam como um dos desafios gerir os limitados recursos públicos disponíveis. Sendo assim, a ES e seus desdobramentos representam uma área interdisciplinar que carece de políticas de incentivo em estudos e pesquisas que desenvolvam novos conhecimentos e práticas de gestão pública a serem implementados no SUS e estimulem o processo de consolidação como área de conhecimento. O presente estudo contribui para reflexão do atual estado da arte da produção científica na área da ES e indica para onde os esforços podem ser direcionados.

\section{REFERÊNCIAS}

1. Almeida AG, Borba JA, Flores LCS. A utilização das informações de custos na gestão da saúde pública: um estudo preliminar em secretarias municipais de saúde do estado de Santa Catarina. Rev. Adm. Pública [online]. 2009; 43 (3): 579-607.

2. Andrade ELG, et al. Pesquisa e produção científica em economia da saúde no Brasil. Revista Administração Pública [online]. 2007; 41 (2): 211-235. 


\section{Gestão em Saúde}

3. Araújo CA. Bibliometria: evolução história e questões atuais. Em Questão. 2006; 12 (1): 11-32.

4. Brasil. Ministério da Saúde. Avaliação econômica em saúde: desafios para gestão no Sistema Único de Saúde. Brasília: Editora do Ministério da Saúde, 2008.

5. Brasil. Ministério da Saúde. Núcleos de Economia da Saúde: orientações para implantação. Série Gestão e Economia da Saúde. v. 1. Brasília: Editora do Ministério da Saúde, 2012.

6. Carias CM, et al. Medicamentos de dispensação excepcional: histórico e gastos do Ministério da Saúde do Brasil. Rev. Saúde Pública [online]. 2011, 45 (2): 233-240.

7. Carvalho G. A saúde pública no Brasil. Estud. av. [online]. 2013; 27 (78): 7-26.

8. Carvalho G. A inconstitucional administração pós-constitucional do SUS através de normas operacionais. Ciência e saúde coletiva [online]. 2001; 6 (2): 435-444.

9. Costa LS, Gadelha CAG, Maldonado J. A perspectiva territorial da inovação em saúde: a necessidade de um novo enfoque. Rev. Saúde Pública [online]. 2012; 46 (suppl.1):59-67.

10. Dain S. Os vários mundos do financiamento da Saúde no Brasil: uma tentativa de integração. Ciênc. saúde coletiva [online]. 2007; 12 (suppl.): 1851-1864.

11. Del Nero CR. O que é economia da saúde. 1995 In: PIOLA, S.F.; VIANNA, S.M. (orgs). Economia da saúde: conceitos e contribuição para a gestão em saúde. Brasília: IPEA, 2002.

12. Gadelha CAG, et al. Saúde e territorialização na perspectiva do desenvolvimento. Ciênc. saúde coletiva [online]. 2011; 16 (6): 3003-3016.

13. Gawryszewski ARB, et al. Acesso ao SUS: representações e práticas de profissionais desenvolvidas nas Centrais de Regulação. Physis [online]. 2012; (22) 1:119-140.

14. Geremia DS. Financiamento do sistema de saúde na Região Metropolitana do Rio de Janeiro: um estudo do município de Nilópolis. Saúde em Debate, Rio de Janeiro, 2011; 35 (91): 522-531.

15. Marinho A. Avaliação da eficiência técnica nos serviços de saúde nos municípios do Estado do Rio de Janeiro. Rev. Bras. Econ. [online]. 2003; 57 (3): 515-534.

16. Mendes A, et al. Discutindo uma Metodologia para a Alocação Equitativa de Recursos Federais para o Sistema Único de Saúde. Saude soc. [online]. 2011; 20 (3): 673-690. 


\section{Gestão em Saúde}

17. Saes SG. Estudo bibliométrico das publicações em economia da saúde no Brasil, 1989 - 1998.115f. Dissertação (Mestrado) - Curso de Pós-Graduação da Faculdade de Saúde Pública da Universidade de São Paulo, 2000.

18. Santos FP, Merhy EE. A regulação pública da saúde no Estado brasileiro: uma revisão. Interface (Botucatu) [online]. 2006; 10 (19): 25-41.

19. Silva L, Rotta CV. O dilema da universalidade e financiamento público do Sistema Único de Saúde no Brasil. Textos \& Contextos (Porto Alegre). 2012; 11(2): 333 - 345.

20. Zucchi P, Del Nero C, Malik AM. Gastos em saúde: os fatores que agem na demanda e na oferta dos serviços de saúde. Saude soc. [online]. 2000; 9 (1-2):127150.

21. Ugá MAD. Sistemas de alocação de recursos a prestadores de serviços de saúde - a experiência internacional. Ciênc. saúde coletiva [online]. 2012; 17 (12): 3437-3445. 